\title{
Funcionalidade de Membro Superior em Mulheres Submetidas ao Tratamento do Câncer de Mama
}

\author{
Upper Limb Functionality in Women after Breast Cancer Treatment
}

Funcionalidad del Miembro Superior en Mujeres Sometidas a Tratamiento del Cáncer de Mama

Elaine Sousa ${ }^{1}$; Flávia Nascimento de Carvalho ${ }^{2}$; Anke Bergmann ${ }^{3}$; Erica Alves Nogueira Fabro ${ }^{4}$; Ricardo de Almeida Dias ${ }^{5}$; Rosalina Jorge Koifman ${ }^{6}$

\section{Resumo}

Introdução: Em decorrência do tratamento do câncer de mama, as mulheres podem evoluir com alteraçôes funcionais, sociais e psicológicas que podem levar à perda de papéis relacionados ao trabalho, família e sexualidade. Objetivo: Avaliar a funcionalidade do membro superior das mulheres submetidas à cirurgia para tratamento do câncer de mama, acompanhadas pelo serviço de fisioterapia em uma instituição pública de referência do Sistema Único de Saúde no Estado do Rio de Janeiro. Método: Estudo transversal em mulheres submetidas a tratamento cirúrgico para câncer de mama. Variáveis relacionadas às características da mulher, do tratamento, do tumor e da funcionalidade do membro superior, mensurada por meio do questionário Disability Arm Shoulder and Hand foram coletadas. Foi realizada uma análise descritiva das variáveis do estudo por meio de medidas de tendência central e de dispersão para as variáveis contínuas e frequências relativa e absoluta para as variáveis categóricas. Resultados: Foram incluídas 105 mulheres que apresentavam idade média de 55,82 anos e índice de massa corporal de 27,80. À inclusão no estudo, a média de tempo transcorrido da cirurgia foi de 1,85 ano. A média do escore total da funcionalidade foi de 27,07 (DP=16,54). Conclusáo: Observou-se um escore satisfatório de capacidade funcional e execução das atividades da vida diária normalmente, contrapondo os estudos realizados por outros autores. Esse resultado positivo deve-se, possivelmente, à atuação da fisioterapia precocemente no tratamento dessas pacientes, comprovando a real necessidade da intervenção fisioterapêutica.

Palavras-chave: Feminino; Neoplasias da Mama-cirurgia; Extremidade Superior-cirurgia; Fisioterapia; Reabilitação; Estudos Transversais

\footnotetext{
Trabalho desenvolvido no Hospital do Câncer III (HC III), Instituto Nacional de Câncer José Alencar Gomes da Silva (INCA).

${ }^{1}$ Fisioterapeuta graduada pelo Centro Universitário Augusto Motta (UNISUAM). Rio de Janeiro (RJ), Brasil. E-mail: elainesline@yahoo.com.br.

${ }^{2}$ Fisioterapeuta. Mestre em Ciências pela Escola Nacional de Saúde Pública Sérgio Arouca (ENSP/Fiocruz). Tecnologista na Coordenação de Prevenção e Vigilância do INCA. Rio de Janeiro (RJ), Brasil. E-mail: fcarvalho@inca.gov.br.

${ }^{3}$ Fisioterapeuta. Doutora em Ciências da Saúde pela ENSP/Fiocruz. Gerente da Divisão de Ensino da Coordenação de Ensino e Divulgação Científica do INCA. Professora-Adjunta e Docente do Programa de Mestrado em Ciências da Reabilitação da UNISUAM. Rio de Janeiro (RJ), Brasil. E-mail: abergmann@inca.gov.br.

${ }^{4}$ Fisioterapeuta. Mestre em Neurociências pela Universidade Federal do Estado do Rio de Janeiro (UNIRIO). Chefe do Serviço de Fisioterapia do Hospital do Câncer III/INCA. Rio de Janeiro, RJ, Brasil. E-mail: efabro@inca.gov.br.

${ }^{5}$ Fisioterapeuta. Especialista em Fisioterapia em Oncologia pela UNISUAM. Fisioterapeuta do Hospital do Câncer III /INCA. Rio de Janeiro (RJ), Brasil.E-mail: ryckdiasfisio@yahoo.com.br.

${ }^{6}$ Médica. Mestre em Medicina Social (Área de Planejamento de Saúde) pela Universidad Nacional Autonoma de Mexico-Xochimico. Doutora em Saúde Pública pela ENSP/Fiocruz. Docente Permanente do Programa de Pós-graduaçâo em Saúde Pública e Meio Ambiente (ENSP/Fiocruz) e do Mestrado em Saúde Coletiva da Universidade Federal do Acre (UFAC). Rio de Janeiro (RJ), Brasil. E-mail: rosajkoifman@gmail.com.

Endereço para correspondência: Rua Marques de Pombal, 125 - Centro. Rio de Janeiro (RJ), Brasil. CEP: 20230-240.
} 


\section{INTRODUÇÃO}

O câncer de mama vem se apresentando como um grande problema de saúde pública, sendo o segundo tipo de câncer mais comum no mundo e o primeiro entre as mulheres ${ }^{1}$. É a principal causa de morte por câncer em mulheres, representando $13,7 \%$ do número de óbitos por câncer no mundo. A sobrevida mundial em cinco anos é de $61 \%{ }^{2,3}$.

No Brasil, são estimados 52.680 novos casos de câncer de mama para 2012 e a mortalidade é bastante elevada, se comparada aos países desenvolvidos, em decorrência do diagnóstico em estadiamento avançado, sendo $80 \%$ dos casos realizados nos estádios III ou IV ${ }^{2}$. Esse fato pode ser devido, entre outros fatores, à ineficácia das estratégias de controle e rastreamento do câncer. A detecção precoce possibilita a utilização de recursos terapêuticos menos agressivos e uma maior probabilidade de cura ${ }^{1,4-5}$.

Atualmente, o tratamento padrão para o câncer de mama em estágios iniciais é composto por cirurgia conservadora e abordagem axilar seguida de radioterapia ${ }^{6}$. Já nos estádios mais avançados, se utiliza o tratamento sistêmico com quimioterapia e a hormonioterapia no sentido de controlar a doença na extensão de todo o organismo 5 .

Durante e após o tratamento oncológico, as mulheres podem evoluir com alteraçóes funcionais, sociais e psicológicas que podem levar à perda de papéis relacionados ao trabalho, família e sexualidade 5 . Todos esses processos podem ser vividos pela paciente e familiares, aumentando os sentimentos relacionados ao sofrimento e à ansiedade ${ }^{3,7}$.

Com o objetivo de minimizar o impacto negativo causado pelo câncer e seu tratamento na qualidade de vida da mulher, a fisioterapia deve ser implementada visando a favorecer o retorno às atividades da vida diária (AVD) e melhor qualidade de vida, fazendo-se necessária em todas as etapas do tratamento do câncer de mama: prétratamento (diagnóstico e avaliação); durante o tratamento (quimioterapia, radioterapia, cirurgia, e hormonioterapia); após o tratamento (período de seguimento); e nos cuidados paliativos. É importante conhecer e identificar as demandas da paciente, os sintomas e suas causas, e o impacto destes nas AVD, em cada uma dessas etapas. A conduta fisioterapêutica deve, então, ser traçada através de orientaçóes domiciliares e tratamentos específicos ${ }^{8}$.

A funcionalidade está relacionada com as atividades desempenhadas na vida diária de cada indivíduo. As complicaçóes decorrentes do tratamento oncológico para o câncer de mama, como: linfedema, dor, parestesia, diminuição da força muscular e redução da amplitude de movimento (ADM) do membro envolvido, merecem atenção, pois podem prejudicar o retorno às atividades rotineiras, comprometendo a qualidade de vida das pacientes ${ }^{8}$.
Este trabalho tem como objetivo avaliar a funcionalidade do membro superior das mulheres submetidas à cirurgia para tratamento do câncer de mama, acompanhadas pelo serviço de fisioterapia em uma instituição pública de referência do Sistema Único de Saúde (SUS) no Estado do Rio de Janeiro, Brasil.

\section{MATERIAL E MÉTODO}

Trata-se de um estudo transversal dentro de uma coorte hospitalar de mulheres submetidas a tratamento cirúrgico para o câncer de mama.

Foram incluídas 105 mulheres submetidas ao tratamento cirúrgico há um ano ou mais, acompanhadas pelo serviço de fisioterapia, na rotina pós-operatória hospitalar.

O protocolo fisioterapêutico inicia-se imediatamente após a cirurgia, à beira do leito, e visa à manutenção dos movimentos de ombro e cuidados com o membro por meio de orientações de exercícios de flexão, abdução, rotação externa de duas a três vezes ao dia e atividades que náo excedam a amplitude de $90^{\circ}$ até a retirada de pontos e dreno. No pré-operatório, essas orientaçōes são, da mesma forma, fornecidas, sendo reforçadas no pós-operatório imediato ${ }^{8}$.

Quando da cicatrização completa da incisão cirúrgica e retirada de dreno, é permitida a execução do arco completo de movimento, entretanto, as recomendaçóes quanto aos movimentos bruscos, repetitivos e com carga, bem como os cuidados com objetos perfuro cortantes, acidentes e traumas, são mantidas nessa fase tardia do pós-operatório com o objetivo de prevenir a manifestação do linfedema de membro superior. As avaliçôes são feitas após 30 dias, 6 meses e 1 ano, caso a paciente não apresente sequelas do tratamento. Caso estas sejam diagnosticadas com quaisquer alterações decorrentes do tratamento oncológico, são encaminhadas ao atendimento fisioterapêutico semanal visando à recuperação da funçáo afetada. Após a alta da fisioterapia, essas pacientes são orientadas a manterem os exercícios e cuidados em domicílio ${ }^{8}$.

Quanto aos critérios, as mulheres com menos de um ano de cirurgia, as que estavam em tratamento adjuvante em curso, aquelas com evoluçâo de doença local ou a distância, as com déficit cognitivo e aquelas com relato de história de alteração funcional em membro superior prévia ao diagnóstico de câncer de mama foram excluídas.

A funcionalidade do membro superior homolateral ao tratamento do câncer de mama foi considerada como variável desfecho, sendo mensurada por meio da aplicação do questionário Disability Arm Shoulder and Hand (DASH), validado na população brasileira'. Esse instrumento contém 30 questōes, com valor máximo de cinco, designadas a medir a função física, sintomas e funçâo social, sendo necessário um mínimo de 27 itens 
respondidos. Para fins de comparabilidade com outras escalas, o escore total foi transformado em uma escala de 100 , subtraindo-se um e multiplicando-se por 25 , onde um escore elevado indica grande incapacidade. O DASH apresenta, ainda, dois módulos opcionais (performance musical ou esportiva e trabalho) contendo quatro itens cada que, por náo corresponderem às características da nossa população, não foram utilizados? .

Foram coletadas as seguintes variáveis descritivas: idade, escolaridade, estado civil, ocupação, índice de massa corporal (IMC), lado dominante, tipo de cirurgia mamária e axilar, tempo transcorrido entre a cirurgia e a avaliação da funcionalidade, estadiamento histopatológico e tratamentos realizados (quimioterapia, radioterapia e hormonioterapia).

Foi realizada uma análise descritiva das variáveis pertencentes ao estudo por meio das medidas de tendência central (média e mediana) e medidas de dispersão (valor mínimo e máximo, desvio-padrão) para as variáveis contínuas e frequência relativa e absoluta, para as variáveis dicotômicas. Para análise dos dados, foi utilizado o pacote estatístico SPSS 17.0 (Statistical Package for the Social Sciences).

Este estudo foi aprovado pelo Comitê de Ética e Pesquisa do Instituto Nacional de Câncer José Alencar Gomes da Silva (INCA) sob o número 84/11. Todas as participantes assinaram um Termo de Consentimento Livre e Esclarecido. A pesquisa foi realizada seguindo todos os pressupostos da resolução CNS 196/06.

\section{RESULTADOS}

Foram incluídas 105 mulheres que apresentavam idade média de 55,82 anos $(\mathrm{DP}=11,61)$ e índice de massa corporal de 27,80 $(\mathrm{DP}=4,30)$. $\mathrm{Na}$ inclusão do estudo, o tratamento cirúrgico do câncer de mama havia sido realizado em média há 1,85 anos $(\mathrm{DP}=1,63)$.

No momento da avaliaçáo fisioterapêutica, a maior parte da população incluída no estudo apresentava 50 anos ou mais $(61,0 \%)$. Eram casadas ou encontravam-se em união estável $48,6 \%$, tinham o segundo grau completo $32,4 \%$, desempenhavam como atividade principal as tarefas do lar 60,0\%. Apresentavam sobrepeso $45,3 \%$ das pacientes e $94,3 \%$ eram destras. O lado dominante foi o mesmo da abordagem cirúrgica em $46,7 \%$ dessas mulheres (Tabela 1).

A avaliação das características tumorais e terapêuticas mostra uma maior prevalência de diagnósticos em estadiamento clínico IIA (25,7\%). Quanto às características cirúrgicas, a maior parte foi submetida à mastectomia com dissecção axilar (66,7\%). A reconstrução mamária imediata foi realizada em 6,2\% das mulheres submetidas à mastectomia. Considerando as modalidades terapêuticas adjuvantes para o câncer de mama, a quimioterapia
Tabela 1. Análise descritiva das variáveis sociodemográficas e clínicas

\begin{tabular}{l|c|c|}
\multicolumn{1}{|c|}{ Variável } & n & $\%$ \\
\hline Idade (anos) & 41 & $39,0 \%$ \\
\hline$<50$ anos & 64 & $61,0 \%$ \\
\hline >ou =50 anos
\end{tabular}

Estado civil

\begin{tabular}{l|c|c}
\hline Casada ou união & 51 & $48,6 \%$ \\
\hline Divorciada/separada & 15 & $14,3 \%$ \\
\hline Viúva & 16 & $15,2 \%$ \\
\hline Solteira & 23 & $21,9 \%$ \\
\hline
\end{tabular}

Nível de escolaridade

\begin{tabular}{l|c|c}
\hline Analfabeto & 2 & $1,9 \%$ \\
\hline Primeiro grau incompleto & 31 & $29,5 \%$ \\
\hline Primeiro grau completo & 22 & $21,0 \%$ \\
\hline Segundo grau incompleto & 3 & $2,9 \%$ \\
\hline Segundo grau completo & 34 & $32,4 \%$ \\
\hline Superior incompleto & 4 & $3,8 \%$ \\
\hline Superior completo & 9 & $8,6 \%$ \\
\hline Ocupação & 63 & $60,0 \%$ \\
\hline Do lar & 42 & $40,0 \%$ \\
\hline Trabalha fora de casa &
\end{tabular}

Status nutricional

\begin{tabular}{l|c|c}
\hline Eutrófico & 26 & $27,4 \%$ \\
\hline Sobrepeso & 43 & $45,3 \%$ \\
\hline Obeso & 26 & $27,4 \%$ \\
\hline Lado dominante & 99 & $94,3 \%$ \\
\hline Direito & 6 & $5,7 \%$ \\
\hline Esquerdo
\end{tabular}

O lado dominante é o lado da cirurgia?

\begin{tabular}{l|c|c}
\hline Sim & 49 & $46,7 \%$ \\
\hline Não & 56 & $53,3 \%$ \\
\hline
\end{tabular}

foi realizada em $52,6 \%$ das mulheres, $70,7 \%$ foram submetidas à hormonioterapia e $76,5 \%$ à radioterapia (Tabela 2).

Ao aplicarmos o questionário para avaliação da funcionalidade (DASH), a média do escore total foi de $27,07(\mathrm{DP}=16,54)($ Figura 1).

As mulheres relataram não haver dificuldades para realizar as seguintes atividades: escrever $(79,0 \%)$, virar uma chave $(94,3 \%)$, preparar uma refeição $(81,9 \%)$, abrir uma porta pesada (39,0\%), colocar algo em uma prateleira acima de sua cabeça $(52,4 \%)$, arrumar a cama $(76,2 \%)$, carregar uma sacola $(69,5 \%)$, trocar uma lâmpada acima de sua cabeça $(47,6 \%)$, lavar ou secar seu cabelo $(88,6 \%)$, lavar suas costas $(61,9 \%)$, vestir uma blusa fechada $(64,8 \%)$, usar uma faca para cortar alimentos 
Tabela 2. Análise descritiva das variáveis tumorais e terapêuticas

\begin{tabular}{|c|c|c|}
\hline Variável & $\mathbf{n}$ & $\%$ \\
\hline \multicolumn{3}{|l|}{ Estadiamento clínico } \\
\hline 0 & 5 & $4,8 \%$ \\
\hline I & 20 & $19,0 \%$ \\
\hline IIA & 27 & $25,7 \%$ \\
\hline IIB & 21 & $20,0 \%$ \\
\hline IIIA & 14 & $13,3 \%$ \\
\hline IIIB & 18 & $17,1 \%$ \\
\hline
\end{tabular}

Tipo de cirurgia + Abordagem axilar

\begin{tabular}{l|c|c}
\hline Mastectomia + BLS & 12 & $11,4 \%$ \\
\hline Mastectomia + LA & 70 & $66,7 \%$ \\
\hline Conservadora + BLS & 17 & $16,2 \%$ \\
\hline Conservadora + LA & 6 & $5,7 \%$ \\
\hline
\end{tabular}

Reconstrução mamária

\begin{tabular}{l|c|c}
\hline Sim & 5 & $6,2 \%$ \\
\hline Não & 76 & $93,8 \%$ \\
\hline Tratamento adjuvante & \multicolumn{2}{|l}{} \\
\hline Quimioterapia adjuvante & 51 & $52,6 \%$ \\
\hline Sim & 46 & $47,4 \%$ \\
\hline Não
\end{tabular}

Radioterapia adjuvante

\begin{tabular}{l|l|l}
\hline Sim & 75 & $76,5 \%$ \\
\hline Não & 23 & $23,5 \%$ \\
\hline
\end{tabular}

Local da radioterapia

\begin{tabular}{l|l|l}
\hline Com axila & 49 & $73,1 \%$ \\
\hline Sem axila & 18 & $26,9 \%$ \\
\hline
\end{tabular}

Hormonioterapia adjuvante

\begin{tabular}{l|l|l}
\hline Sim & 70 & $70,7 \%$ \\
\hline Não & 29 & $29,3 \%$ \\
\hline
\end{tabular}

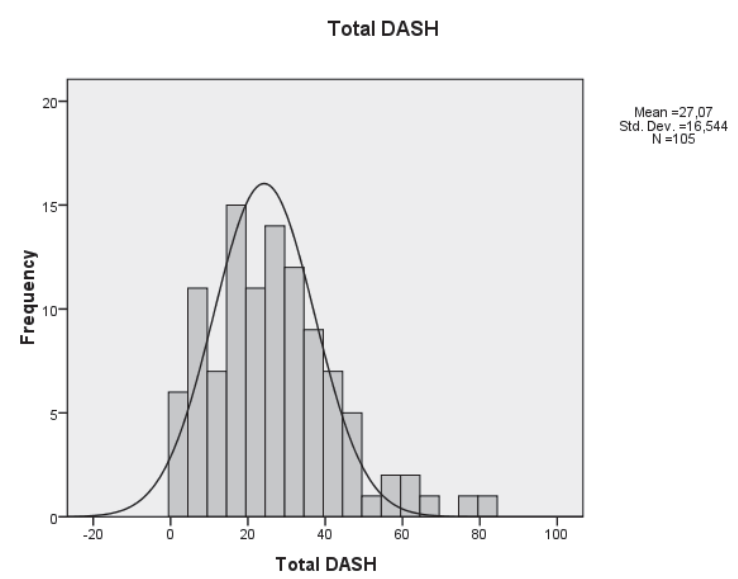

Figura 1. Escore do DASH no momento da avaliação fisioterapêutica
(72,4\%), participar de atividades recreativas que exijam pouco esforço $(77,1 \%)$, realizar atividades recreativas nas quais mova seu braço livremente $(70,5 \%)$, transportar-se um lado para outro $(87,6 \%)$ e praticar atividades sexuais $(54,4 \%)$ (Tabela 3).

As mulheres relataram não conseguir realizar tarefas pesadas em $51,4 \%$ dos casos, exercer trabalho de jardinagem em 49,0\%, carregar um objeto pesado em $42,9 \%$, participar de atividades recreativas que exijam força ou impacto nos braços, ombro ou mãos $(41,0 \%)$ e abrir um vidro novo com uma tampa muita apertada (29,5\%) (Tabela 3).

Quanto ao quadro álgico, 48,6\% das pacientes negaram sensação dolorosa no membro superior homolateral ao tratamento cirúrgico do câncer de mama ao realizar alguma atividade específica e $54,3 \%$ das mulheres negaram a sensação de dor espontânea. Em $54,3 \%$ dos casos, não foi observado desconforto na pele, $59,0 \%$ negaram fraqueza no braço, ombro ou mão; e $64,8 \%$ das mulheres apresentaram dificuldade para mover o membro superior (Tabela 4).

Quando questionadas em que ponto o seu problema com braço, ombro ou mão afetou suas atividades normais com família, amigos, vizinhos ou colegas, ou se o seu trabalho ou atividades diárias normais foram limitados devido ao seu problema com braço, ombro ou mão, $72,4 \%$ relataram que náo afetou, $52,4 \%$ não limitou (Tabela 5). Em nosso estudo, $72,4 \%$ das mulheres relataram que a gravidade dos sintomas no membro não afetou suas atividades com terceiros e $52,4 \%$ das pacientes náo se sentiram limitadas para realizarem atividades laborais ou diárias.

\section{DISCUSSÃO}

Este estudo incluiu mulheres com câncer de mama, tratadas em uma instituição pública de referência, onde a abordagem fisioterapêutica se dá durante todas as fases do tratamento oncológico ${ }^{8}$.

Em relação à idade, estado civil e escolaridade, as mulheres estudadas apresentam perfil similar aos achados dos estudos publicados por outros autores. Quanto ao estadiamento clínico ao diagnóstico, foi identificado em nosso estudo que a maior parte das mulheres apresentava-se em estádios avançados da doença (50,4\% maior ou igual a II B), o que também tem sido observado em outros estudos realizados na populaçáo brasileira ${ }^{4,10-13}$.

Nicolussi et al. ${ }^{14}$ afirmaram que as mulheres vivenciam tanto problemas físicos e emocionais, como problemas sociais com suas famílias e em suas atividades diárias devido ao câncer de mama e seu tratamento. Alguns autores relataram um maior impacto das alteraçóes emocionais, quando comparadas com as físicas ${ }^{7,15}$. Esses dados não correspondem aos achados de nosso estudo, em 
Tabela 3. Escore do DASH de acordo com a habilidade em fazer as atividades

\begin{tabular}{|c|c|c|c|c|c|}
\hline \multirow{2}{*}{$\begin{array}{c}\text { Meça a sua habilidade em fazer as } \\
\text { seguintes atividades na semana } \\
\text { passada }\end{array}$} & $\begin{array}{l}\text { Não houve } \\
\text { dificuldade }\end{array}$ & $\begin{array}{c}\text { Houve } \\
\text { pouca } \\
\text { dificuldade }\end{array}$ & $\begin{array}{l}\text { Houve } \\
\text { dificuldade } \\
\text { média }\end{array}$ & $\begin{array}{c}\text { Houve } \\
\text { muita } \\
\text { dificuldade }\end{array}$ & $\begin{array}{l}\text { Não } \\
\text { conseguiu } \\
\text { fazer }\end{array}$ \\
\hline & n (\%) & n (\%) & n (\%) & n (\%) & n (\%) \\
\hline $\begin{array}{l}\text { Abrir um vidro novo com uma tampa } \\
\text { muita apertada }\end{array}$ & $30(28,6 \%)$ & $13(12,4 \%)$ & $16(15,2 \%)$ & $15(14,3 \%)$ & $31(29,5 \%)$ \\
\hline Escrever & $83(79,0 \%)$ & $6(5,7 \%)$ & $9(8,6 \%)$ & $6(5,7 \%)$ & $1(1,0 \%)$ \\
\hline Virar uma chave & $99(94,3 \%)$ & $1(1,0 \%)$ & $4(3,8 \%)$ & $1(1,0 \%)$ & --- \\
\hline Preparar uma refeição & $86(81,9 \%)$ & $11(10,5 \%)$ & $4(3,8 \%)$ & $3(2,9 \%)$ & $1(1,0 \%)$ \\
\hline Abrir uma porta pesada & $41(39,0 \%)$ & $11(10,5 \%)$ & $11(10,5 \%)$ & $15(14,3 \%)$ & $27(25,7 \%)$ \\
\hline $\begin{array}{l}\text { Colocar algo em uma prateleira acima } \\
\text { de sua cabeça }\end{array}$ & $55(52,4 \%)$ & $16(15,2 \%)$ & $11(10,5 \%)$ & $13(12,4 \%)$ & $10(9,5 \%)$ \\
\hline $\begin{array}{l}\text { Fazer tarefas domésticas pesadas (por } \\
\text { exemplo: lavar paredes, lavar o chão) }\end{array}$ & $18(17,1 \%)$ & $8(7,6 \%)$ & $12(11,4 \%)$ & $13(12,4 \%)$ & $54(51,4 \%)$ \\
\hline Fazer trabalho de jardinagem & $37(37,0 \%)$ & $1(1,0 \%)$ & $8(8,0 \%)$ & $4(4,0 \%)$ & $49(49,0 \%)$ \\
\hline Arrumar a cama & $80(76,2 \%)$ & $13(12,4 \%)$ & $8(7,6 \%)$ & $4(3,8 \%)$ & --- \\
\hline Carregar uma sacola ou uma maleta & $73(69,5 \%)$ & $8(7,6 \%)$ & $12(11,4 \%)$ & $8(7,6 \%)$ & $4(3,8 \%)$ \\
\hline $\begin{array}{l}\text { Carregar um objeto pesado (mais de } \\
5 \mathrm{~kg} \text { ) }\end{array}$ & $25(23,8 \%)$ & $8(7,6 \%)$ & $8(7,6 \%)$ & $19(18,1 \%)$ & $45(42,9 \%)$ \\
\hline Trocar uma lâmpada acima da cabeça & $50(47,6 \%)$ & $10(9,5 \%)$ & $4(3,8 \%)$ & $7(6,7 \%)$ & $34(32,4 \%)$ \\
\hline Lavar ou secar o cabelo & $93(88,6 \%)$ & $7(6,7 \%)$ & $3(2,9 \%)$ & $2(1,9 \%)$ & -- \\
\hline Lavar suas costas & $65(61,9 \%)$ & $19(18,1 \%)$ & $7(6,7 \%)$ & $8(7,6 \%)$ & $6(5,7 \%)$ \\
\hline Vestir uma blusa fechada & $68(64,8 \%)$ & $17(16,2 \%)$ & $10(9,5 \%)$ & $10(9,5 \%)$ & $-\cdots$ \\
\hline Usar uma faca pra cortar alimentos & $76(72,4 \%)$ & $13(12,4 \%)$ & $6(5,7 \%)$ & $7(6,7 \%)$ & $3(2,9 \%)$ \\
\hline $\begin{array}{l}\text { Atividades recreativas que exigem } \\
\text { pouco esforço (jogar cartas, tricotar) }\end{array}$ & $81(77,1 \%)$ & $4(3,8 \%)$ & $4(3,8 \%)$ & $3(2,9 \%)$ & $13(12,4 \%)$ \\
\hline $\begin{array}{l}\text { Atividades recreativas que exigem } \\
\text { força ou impacto nos braços, ombros } \\
\text { ou mãos (jogar vôlei, martelar) }\end{array}$ & $38(36,2 \%)$ & $3(2,9 \%)$ & $9(8,6 \%)$ & $12(11,4 \%)$ & $43(41,0 \%)$ \\
\hline $\begin{array}{l}\text { Atividades recreativas nas quais você } \\
\text { move seu braço livremente (como: } \\
\text { pescar, jogar) }\end{array}$ & $74(70,5 \%)$ & $7(6,7 \%)$ & $7(6,7 \%)$ & $9(8,6 \%)$ & $8(7,6 \%)$ \\
\hline $\begin{array}{l}\text { Transportar-se de um lado para outro } \\
\text { (ir de um lugar a outro) }\end{array}$ & $92(87,6 \%)$ & $1(1,0 \%)$ & $6(5,7 \%)$ & $6(5,7 \%)$ & --- \\
\hline Atividades sexuais & $56(54,4 \%)$ & $3(2,9 \%)$ & $7(6,8 \%)$ & $2(1,9 \%)$ & $35(34,0 \%)$ \\
\hline
\end{tabular}

Tabela 4. Escore do DASH de acordo com a gravidade dos sintomas

\begin{tabular}{l|c|c|c|c|c}
\hline \multirow{2}{*}{$\begin{array}{c}\text { Meça a gravidade dos seguintes } \\
\text { sintomas na semana passada }\end{array}$} & Nenhuma & Pouca & Mediana & Muita & Extrema \\
\cline { 2 - 6 } & $\mathbf{n}(\%)$ & $\mathbf{n}(\%)$ & $\mathbf{n}(\%)$ & $\mathbf{n}(\%)$ & $\mathbf{n}(\%)$ \\
\hline Dor no braço, ombro ou mão & $51(48,6 \%)$ & $19(18,1 \%)$ & $22(21,0 \%)$ & $11(10,5 \%)$ & $2(1,9 \%)$ \\
\hline $\begin{array}{l}\text { Dor no braço, ombro ou mão quando } \\
\text { você fazia alguma atividade espeć́fica }\end{array}$ & $57(54,3 \%)$ & $14(13,3 \%)$ & $19(18,1 \%)$ & $10(9,5 \%)$ & $5(4,8 \%)$ \\
\hline $\begin{array}{l}\text { Desconforto na pele (alfinetadas) no } \\
\text { braço, ombro ou mão }\end{array}$ & $57(54,3 \%)$ & $14(13,3 \%)$ & $21(20,0 \%)$ & $9(8,6 \%)$ & $4(3,8 \%)$ \\
\hline Fraqueza no braço, ombro ou mão & $62(59,0 \%)$ & $18(17,1 \%)$ & $14(13,3 \%)$ & $8(7,6 \%)$ & $3(2,9 \%)$ \\
\hline $\begin{array}{l}\text { Dificuldade em mover braço, ombro } \\
\text { ou mão }\end{array}$ & $68(64,8 \%)$ & $11(10,5 \%)$ & $14(13,3 \%)$ & $8(7,6 \%)$ & $4(3,8 \%)$ \\
\hline
\end{tabular}


Tabela 5. Escore do DASH de acordo com a gravidade dos sintomas

\begin{tabular}{|l|c|c|c|c|c}
\hline $\begin{array}{c}\text { Meça a gravidade dos } \\
\text { seguintes sintomas na semana } \\
\text { passada }\end{array}$ & $\begin{array}{c}\text { Não } \\
\text { afetou }\end{array}$ & $\begin{array}{c}\text { Afetou } \\
\text { pouco }\end{array}$ & $\begin{array}{c}\text { Afetou } \\
\text { medianamente }\end{array}$ & $\begin{array}{c}\text { Afetou } \\
\text { muito }\end{array}$ & $\begin{array}{c}\text { Afetou } \\
\text { extremamente }\end{array}$ \\
\cline { 2 - 6 } & $\mathbf{n}(\%)$ & $\mathbf{n}(\%)$ & $\mathbf{n}(\%)$ & $\mathbf{n}(\%)$ & $\mathbf{n}(\%)$ \\
\hline $\begin{array}{l}\text { Na semana passada, em que } \\
\text { ponto o seu problema com braço, } \\
\text { ombro ou mão afetou suas } \\
\text { atividades normais com família, } \\
\text { amigos, vizinhos ou colegas? }\end{array}$ & $76(72,4 \%)$ & $10(9,5 \%)$ & $9(8,6 \%)$ & $7(6,7 \%)$ & $3(2,9 \%)$ \\
\hline $\begin{array}{c}\text { Meça a gravidade dos } \\
\text { seguintes sintomas na semana } \\
\text { passada }\end{array}$ & $\begin{array}{c}\text { Não } \\
\text { limitou }\end{array}$ & $\begin{array}{c}\text { Limitou } \\
\text { pouco }\end{array}$ & $\begin{array}{c}\text { Limitou } \\
\text { medianamente }\end{array}$ & $\begin{array}{c}\text { Limitou } \\
\text { muito }\end{array}$ & extremamente \\
Limitou
\end{tabular}

que a maioria das pacientes náo relacionou o problema no membro e comprometimento de suas relaçóes com terceiros, nem de suas atividades laborais.

Quanto às alteraçóes físicas, no que diz respeito ao relato de dor, 51,6\% relataram presença de dor, sendo que a dor intensa foi menos prevalente. As pacientes retomaram suas AVD normalmente, sendo possível identificar, quando aplicado o questionário DASH, que estas relataram não ter dificuldades na maioria das atividades descritas. Lamino et al. ${ }^{11}$ relataram ocorrência de dor em 47,2\% das mulheres estudadas, sendo classificada como moderada e intensa, o que também é encontrado no estudo de Fabro et al. ${ }^{16}$, em que 52,9\% das mulheres estudadas apresentaram síndrome dolorosa e 52,6\% se queixaram de hiperestesia após a cirurgia. Magaldi et al. ${ }^{17}$ referem que, além da dor crônica, outras complicaçóes podem ocorrer após o tratamento do câncer de mama, como os distúrbios sensoriais, edema e alteração na amplitude de movimento que podem persistir por $2 \mathrm{a}$ 5 anos após a cirurgia.

A literatura relata diversas dificuldades físicas relacionadas à terapêutica do câncer de mama, entre elas, as alteraçóes na funcionalidade do membro superior das mulheres que interferem diretamente na qualidade de vida após a cirurgia $^{10,18-20}$. Embora náo tendo encontrado os mesmos achados para a alteraçáo da mobilidade e da sensibilidade do membro em nosso estudo, a prevalência de incapacidade para executar as atividades que exigem grandes esforços com o braço pode refletir o fato de as pacientes terem acompanhamento fisioterapêutico desde o diagnóstico do câncer de mama, conforme rotina institucional, com recomendaçôes para evitar grandes esforços ${ }^{8}$.

Devido à alta incidência e prevalência de complicaçôes decorrentes do tratamento do câncer de mama, diferentes autores concordam no que diz respeito à importância da atuaçáo da fisioterapia o mais precoce possível, devendo esta ser realizada durante todas as fases do tratamento do câncer de mama ${ }^{8,17,21}$. Em estudo transversal realizado com o objetivo de demonstrar o reconhecimento dos recursos fisioterapêuticos em pacientes oncológicos, $23 \%$ dos fisioterapeutas entrevistados relataram acreditar que o tratamento melhora a qualidade de vida, $13,5 \%$ afirmaram sua ação na melhora na funcionalidade e independência nas AVD e 9,5\% relataram ação preventiva e terapêutica eficaz nas complicaçóes oncológicas ${ }^{22}$.

Button et al. ${ }^{23}$, em estudo observacional prospectivo, encontram piores escores de funcionalidade em mulheres submetidas à reconstrução mamária com retalho miocutâneo do grande dorsal, principalmente nos três primeiros meses de pós-operatório. Em um ensaio clínico randomizado, piloto, Campbell et al. ${ }^{24}$ avaliaram o benefício dos exercícios para mulheres com diagnóstico de câncer de mama em estadiamento precoce, submetidas à terapia adjuvante e observaram que, após 12 semanas, $o$ grupo de exercícios apresentou níveis significativamente maiores de funçáo física e elevados escores de qualidade de vida em relação aos controles. Tais achados ratificam a necessidade da intervenção fisioterapêutica em todos os níveis, principalmente, na prevenção das sequelas do tratamento oncológico. 


\section{CONCLUSÃO}

Observou-se que as pacientes encontravam-se com um grau satisfatório no que diz respeito à capacidade funcional e exercem suas AVD sem dificuldade. Esse resultado pode ocorrer devido à atuação da fisioterapia precocemente no tratamento dessas pacientes, comprovando, assim, a real necessidade da intervenção da fisioterapia.

\section{CONTRIBUIÇÕES}

Elaine Sousa, Erica Alves Nogueira Fabro e Ricardo de Almeida Dias contribuíram na obtenção, análise e interpretação dos dados; na redação e revisão crítica. Flávia Nascimento de Carvalho, Anke Bergmann, Rosalina Jorge Koifman contribuíram na concepção e planejamento do projeto de pesquisa; na obtenção, análise e interpretação dos dados; na redação e revisão critica.

\section{Declaraçáo de Conflito de Interesses: Nada a Declarar.}

\section{REFERÊNCIAS}

1. Batiston AP, Tamaki EM, Souza LA SM, Santos MLM. Conhecimento e prática sobre os fatores de risco para o câncer de mama entre mulheres de 40 a 69 anos. Rev. bras. saúde matern. infant. 2011; 11(2):163-71.

2. Instituto Nacional de Câncer José Gomes Alencar da Silva. Estimativa 2012: incidência de câncer no Brasil. Rio de Janeiro: INCA; 2011. 118 p.

3. Cintra JRD, Teixeira MTB, Diniz RW, Gonçalves Júnior $\mathrm{H}$, Florentino TM, Freitas GF, et al. Perfil histoquímico e variáveis clinicopatológicas no câncer de mama. Rev Assoc Med Bras. (1992). 2012; 58(2):178-87.

4. Gonçalves LLC, Santos SB, Marinho EC, Almeida AM, Santos AHS, Barros AMMS, et al. Câncer de mama feminino: aspectos clínicos e patológicos dos casos cadastrados de 2005 a 2008 num serviço público de oncologia de Sergipe Rev. bras saúde matern infant. 2012; 12(1):47-54.

5. Majewski JM, Lopes ADF, Davoglio T, Leite JCC. Qualidade de vida em mulheres submetidas à mastectomia comparada com aquelas que se submeteram à cirurgia conservadora : uma revisão de literatura. Ciên saúde coletiva 2012; 17 (3):707-16.

6. Marta GN, Hanna SA, Martella E, Silva JLF, Carvalho HA. Câncer de mama estádio inicial e radioterapia: atualização. Rev Assoc Med Bras. (1992) 2011; 57 (4):468-74.

7. Silva G, Santos MA. Estressores pós-tratamento do câncer de mama : um enfoque qualitativo. Rev. latinoam enferm. 2010; 18(4): [08 telas].

8. Bergmann A, Ribeiro MJP, Pedrosa E, Nogueira EA, Oliveira ACG. Fisioterapia em mastologia oncológica : rotinas do Hospital do Câncer III/INCA. Rev bras cancerol. 2006; 52(1):97-109.

9. Orfale AG, Araújo PMP, Ferraz MB, Natour J. Translation into Brazilian Portuguese, cultural adaptation and evaluation of the reliability of the disabilities of the arm, shoulder and hand questionnaire. Braz $\mathrm{j}$ med biol res [Internet]. 2005 [acesso 2013 jan 03]; 38(2):293302. Disponível em: http://www.ncbi.nlm.nih.gov/ pubmed/15785841.

10. Santos MSM, Panobianco MS, Mamede MV, Meirelles MCCC, Barros VMB. Sensibilidade tátil no membro superior de mulheres submetidas à linfonodectomia axilar por câncer de mama. Revista brasil ginecol obstetr. 2009; 31(17):361-6.

11. Lamino DA, Mota DDCF, Pimenta CAM. Prevalência e comorbidade de dor e fadiga em mulheres com câncer de mama. Rev Esc Enf USP 2011; 45(2):508-14.

12. Macchetti AH. Estadiamento do câncer de mama diagnosticado. Medicina (Ribeirão Preto) 2007; 40(3):394-402.

13. Martins LC, Ferreira Filho C, Del Giglio A, Munhões DA, Trevizan LLB, Herbst LG, et al. Desempenho profissional ou doméstico das pacientes em quimioterapia para câncer de mama. Rev Assoc Med Bras (1992). 2009; 55(2):158-62.

14. Nicolussi AC, Sawada NO. Qualidade de vida de pacientes com câncer de mama em terapia adjuvante. Rev gaúch enferm. 2011; 32(4):759-66.

15. Rossi L, Santos MA. Repercussōes psicológicas do adoecimento e tratamento em mulheres acometidas pelo câncer de mama. Psicol ciên prof. 2003; 23(4):32-41.

16. Fabro EAN, Bergmann A, Silva BA, Ribeiro ACP, Abrahão KS, Ferreira MGCL, et al. Post-mastectomy pain syndrome: incidence and risks. Breast [Internet]. 2012 [acesso $2012 \mathrm{dez}$ 1]; 21(3):321-5. Disponível em: http://www.ncbi.nlm.nih.gov/pubmed/22377590.

17. Magaldi CM, Barros ACSD, Magaldi FM, Mantese JC, Pinotti JA. Avaliação da morbidade e funcionalidade do membro superior em mulheres submetidas à linfadenectomia axilar total e biópsia de linfonodo sentinela por câncer de mama. Rev bras mastologia. 2005; 15(1):9-14.

18. Sant'Anna DK, Almeida V, Petito EL, Gutiérrez MGR. Adesão à prática de exercícios para reabilitação funcional de mulheres com câncer de mama: revisão de literatura Ciencia y Enfermeria 2010; 16(1):97-104.

19. Gärtner R, Jensen MB, Nielsen J, Ewertz M, Kroman $\mathrm{N}$, Kehlet H. Prevalence of and factors associated with persistent pain following breast cancer surgery. JAMA 2009; 302(18):1985-92. Erratum in: JAMA. 2012; 308(19):1973

20. Hack TF, Kwan WB, Thomas-Maclean RL, Towers A, Miedema B, Tilley A, et al. Predictors of arm morbidity following breast cancer surgery. Psychooncology, 2010; 19(11):1205-12. 
21. Instituto Nacional de Câncer (Brasil). Controle do câncer de mama: documento de consenso. Rio de Janeiro: INCA; 2004. 33p.

22. Borges CAM, Silveira CF, Lacerda PCMT, Nascimento MTA. Análise dos métodos de avaliação, dos recursos e do reconhecimento da fisioterapia oncológica nos hospitais públicos do Distrito Federal. Rev bras cancerol. 2008; 54(4):333-44.

23. Button J, Scott J, Taghizadeh R, Weiler-Mithoff E, Hart AM. Shoulder function following autologous latissimus dorsi breast reconstruction. A prospective three year observational study comparing quilting and non-quilting donor site thechniques. J Plast Reconstr Aesthet Surg. 2010; 63(9):1505-12. Epub 2009 Oct 12

24. Campbell A, Mutrie N, White F, McGuire F, Kearney N. A pilot study of a supervised group exercise programme as a rehabilitation treatment for wonen with breast cancer receiving adjuvant treatment. Eur J Oncol Nurs. 2005; 9(1):56-63. 


\begin{abstract}
Introduction: As a result of the breast cancer treatment, women can develop functional, social and psychological alterations that can lead to loss of roles related to work, family and sexuality. Objective: To evaluate the functionality of the upper limb of women undergoing surgery for breast cancer, followed by a physiotherapy service in a public unit which is a reference in the Unified Health System in the State of Rio de Janeiro. Method: Cross-sectional study in women who were subjected to surgical treatment for breast cancer. Variables related to the characteristics of women, treatment, tumor and functionality of the upper limb, measured by the questionnaire Disability Arm Shoulder and Hand were collected. We performed a descriptive analysis of the study variables using measures of central tendency and dispersion for continuous variables and absolute and relative frequencies for categorical variables. Results: We included 105 women with a mean age of 55.82 years and a body mass index of 27.80. At study entry, the average elapsed time of surgery was 1.85 years. The average of the total score of functionality was $27.07(\mathrm{SD}=16.54)$. Conclusion: There was a satisfactory score of functional capacity and performance of common activities of daily living, contrasting with studies written by other authors. This positive result is possibly due to the role of physiotherapy in the beginning of the treatment of these patients, proving the real need of physiotherapy intervention.

Key words: Female; Breast Neoplasms-surgery; Upper Extremity-surgery; Physical Therapy Specialty; Rehabilition; Cross-Sectional Studies
\end{abstract}

\title{
Resumen
}

Introducción: En consecuencia del tratamiento del cáncer de mama, las mujeres pueden evolucionar con alteraciones funcionales, sociales y psicológicas que pueden llevar a la perdida de las funciones relacionadas al trabajo, familia y sexualidad. Objetivo: Evaluar la funcionalidad del miembro superior en las mujeres sometidas a cirugía para tratamiento del cáncer de mama con seguimiento del servicio de fisioterapia en una institución pública de referencia del Sistema Nacional de Salud en el Estado de Río de Janeiro. Método: Estudio transversal en mujeres sometidas a cirugía para el cáncer de mama. Variables relacionadas a las características de la mujer, del tratamiento, del tumor y, de la funcionalidad del miembro superior mensurada a través de encuesta Discapacidad Hombro, Brazo y Mano han sido recogidas. Fue realizado un análisis descriptivo de las variables del estudio a través de medidas de tendencia central y dispersión para las variables continuas y frecuencias relativas y absolutas para las variables categóricas. Resultados: Han sido incluidas 105 mujeres que presentaban un promedio de edad de 55,82 años y un índice de masa corporal de 27,80. Al ingresar en el estudio, el promedio de tiempo transcurrido de la cirugía fue de 1,85 años. El promedio de la puntuación total de la funcionalidad total fue de 27,07 (DP=16.54). Conclusión: Se ha observado una puntuación satisfactoria de la capacidad funcional y ejecución de las actividades de la vida diaria en general, contraponiendo los estudios realizados por otros autores. Este resultado positivo se debe posiblemente a la actuación de la fisioterapia precoz en el tratamiento de esos pacientes, comprobando la real necesidad de intervención fisioterapéutica.

Palabras clave: Femenino; Neoplasias de la Mama-cirugía; Extremidad Superior-cirurgia; Fisioterapia; Rehabilitación; Estudios Transversales 